\title{
Bose-Einstein Condensates with Large Number of Vortices
}

\author{
Tin-Lun Ho \\ Department of Physics, The Ohio State University, Columbus, Ohio 43210
}

\begin{abstract}
We show that as the number of vortices in a three dimensional Bose-Einstein Condensate increases, the system reaches a "quantum Hall" regime where the density profile is a Gaussian in the $x y$-plane and an inverted parabolic profile along $z$. The angular momentum of the system increases as the vortex lattice shrinks. However, Coriolis force prevents the unit cell of the vortex lattice from shrinking beyond a minimum size. Although the recent MIT experiment is not exactly in the quantum Hall regime, it is close enough for the present results to be used as a guide. The quantum Hall regime can be easily reached by moderate changes of the current experimental parameters.
\end{abstract}

In a recent experiment [1] , the MIT group led by Wolfgang Ketterle has generated as many as 160 vortices in a Bose-Einstein condensate of $\mathrm{Na}$ atoms. While the vortices form a hexagonal lattice as expected for equilibrium systems, many observed properties appear to differ significantly from the predicted ones [1]. It is thought that the system may not be in equilibrium.

Equilibrium or not, it is clear from the recent Paris [2] and MIT experiments [1] that one can deposit a large amount of angular momentum to the condensate by driving it with an asymmetric potential at a rotation frequency $\Omega_{d}$ close to the quadrupolar resonance. In view of the recent rapid developments [1] [2], it is conceivable that higher, or even much higher angular momentum states can be achieved in the near future. The high angular momentum states are interesting because they resemble some of the novel solid state systems such as type-II superconductor and quantum Hall liquid. However, the unique features of Bose-Einstein condensates also introduce important differences.

The purpose of this paper is discuss the properties of Bose-Einstein condensates with large number of vortices. We show that the physics in this regime is closely related to two dimensional quantum Hall physics, even though the system is three dimensional. The specific wavefunction in this regime allows one to calculate many properties of the system, including the effective rotational frequency $\Omega=\partial E / \partial L$. It is clear that $\Omega$ will not be the same as the driving frequency $\Omega_{d}$, since it depends only on the total angular momentum $L$, which can be varied by varying the duration of the drive, even though $\Omega_{d}$ is held fixed. The fact that the vortex lattice has the equilibrium form strongly suggests that the system is in quasi-equilibrium, characterized by an $L$ and $\Omega$ which change slowly because of the residual asymmetry in the confining potential. It is conceivable that some of the discrepancy with theory mentioned in ref. 11 might disappear once the effective frequency $\Omega$ is identified.

We shall see that in the quantum Hall regime, Corio- lis force prevents the unit cell $(v)$ of the vortices lattice from shrinking below $v^{*}=\pi a_{\perp}^{2}$ where $a_{\perp}$ is the oscillator length in the $x y$-plane. For a large condensate, the density profile along $z$ is an inverted parabola similar to the stationary case. However, it is a Gaussian in the $x y$ plane, with a width $\sigma$ that scales as $R_{z}^{-3 / 2}$, where $R_{z}$ is the maximum extent of the condensate along $z$. The angular momentum of the system is proportional to $\pi \sigma^{2}$, which grows as the size of unit cell $v$ shrinks.

The quantum Hall regime and the minimum vortex separation: The condensate wavefunction $\Psi$ is determined by the Gross-Pitaevskii functional

$$
\mathcal{K}=\int \Psi^{*}\left[h_{z}+h_{\perp}-\Omega L_{z}-\mu\right] \Psi+\frac{1}{2} g \int|\Psi|^{4},
$$

where $h_{z}=-\frac{\hbar^{2}}{2 M} \nabla_{z}^{2}+\frac{1}{2} M \omega_{z}^{2} z^{2}$ and $h_{\perp}=-\frac{\hbar^{2}}{2 M} \nabla_{\perp}^{2}+$ $\frac{1}{2} M \omega_{\perp}^{2} r^{2}$ are the single particle Hamiltonians along $z$ and in the $x y$-plane, $\omega_{z}$ and $\omega_{\perp}$ are the frequencies of the harmonic potentials in $z$ and in the $x y$-plane; $\mathbf{r} \equiv(x, y), L_{z}=-i \hbar \hat{\mathbf{z}} \cdot \mathbf{r} \times \nabla$ is the angular momentum; $g=4 \pi \hbar^{2} a_{s c} / M, a_{s c}$ is the s-wave scattering length, and $(\Omega, N)$ are Lagrange multipliers specifying the angular momentum and particle number of the system. It is useful to rewrite $h_{\perp}-\Omega L_{z}=\mathcal{H}_{L}+\hbar\left(\omega_{\perp}-\Omega\right) L_{z}$, where

$$
\mathcal{H}_{L}=\left(-i \hbar \nabla_{\perp}-M \omega_{\perp} \hat{\mathbf{z}} \times \mathbf{r}\right)^{2} /(2 M)
$$

Eq.(2) is precisely the Hamiltonian $\frac{1}{2 M}(-i \hbar \nabla-e \mathbf{A} / c)^{2}$ of a charge $-e$ particle moving in $x y$-plane subjected to a magnetic field $B \hat{\mathbf{z}}$ with a vector potential $\mathbf{A}=\frac{1}{2} B \hat{\mathbf{z}} \times \mathbf{r}$, $e B / M c=2 \Omega$. The eigenfunctions of $\mathcal{H}_{L}$ are

$$
h_{n, m}(\mathbf{r})=\frac{e^{|u|^{2} /\left(2 a_{\perp}^{2}\right)} \partial_{+}^{m} \partial_{-}^{n} e^{-|u|^{2} / a_{\perp}^{2}}}{\sqrt{\pi a_{\perp}^{2} n ! m !}}
$$

with eigenvalues $\epsilon_{n, m}=\hbar \omega_{\perp}(2 n+1)$ where $n$ and $m$ are integers $0,1,2, . ., u \equiv(x+i y) / a_{\perp}, a_{\perp}=\sqrt{\hbar / M \omega_{\perp}}$, $\partial_{ \pm} \equiv\left(a_{\perp} / 2\right)\left(\partial_{x} \pm i \partial_{y}\right)$. The integer $n$ is referred to as the Landau level index, and $m$ labels the degenerate states within a Landau level $n$. Since $u_{n, m}$ are also eigenstates of $L_{z}=\hbar\left(u \partial_{u}-u^{*} \partial_{u^{*}}\right)$ with eigenvalue $\hbar(m-n)$. The eigenvalue of $h_{\perp}-\Omega L_{z}$ is $\hbar\left[\left(\omega_{\perp}+\Omega\right) n+\left(\omega_{\perp}-\Omega\right) m+\right.$ $\left.\omega_{\perp}\right]$. The degeneracy of the Landau level is lifted by the deviation $\omega_{\perp}-\Omega$. [3]

Interaction effects will mix different $(n, m)$ states. For sufficiently small $g$ and $\omega_{\perp}-\Omega$, the system primarily resides in the lowest Landau level (LLL), $(n=0)$, since other levels are separated from it by an energy gap $\geq \hbar \omega_{\perp}$. At first sight, this might appear to be difficult to achieve for it requires $g n<<\hbar \omega_{\perp}$, which implies either $g$ is exceedingly small or density the $n$ is very low 
[4]. While this is true for low angular momentum states, it is not true in the high angular momentum limit. As we shall see, as the number of vortices increases, (corresponding to $\Omega$ very close to $\omega_{\perp}$ ), the density is thinned out sufficiently in the $x y$-plane so that the LLL regime can be attained. We shall therefore proceed by assuming that the system in the LLL, and return later to show that mixing of higher Landau level is indeed small in the fast rotating limit for large condensates with typical interactions.

Before proceeding, we note that since the dynamics along $z$ is identical to the non-rotating case, we can apply the usual Thomas-Fermi approximation along $z$ to ignore the kinetic energy $\left|\nabla_{z} \Psi\right|^{2}$ term and justify it at the end. Our task is then to minimize $K=\int \mathrm{d} z \mathcal{K}(z)$,

$$
\mathcal{K}(z)=\int \mathrm{d} \mathbf{r} \Psi^{*}\left[h_{\perp}-\Omega L_{z}-\mu(z)\right] \Psi+\frac{1}{2} g \int|\Psi|^{4}
$$

with the constraint $N=\int|\Psi|^{2}$, where $\mu(z)=\mu-$ $\frac{1}{2} M \omega_{z}^{2} z^{2}$. Since $u_{0, m}(\mathbf{r}) \propto u^{m} e^{-r^{2} / 2 a_{\perp}}$, a general wavefunction function in the lowest Landau level is $\phi(\mathbf{r})=$ $f(u) e^{-r^{2} / 2 a_{\perp}}$, where $f(u)$ is an analytic function of $u$. Because of the Guassian cutoff, it is sufficient to take $f$ as a polynomial of power $Q$, which can be written in a factorized form $\prod_{\alpha=1}^{Q}\left(u-b_{\alpha}\right)$ according to the fundamental theorem of algebra, where $b_{\alpha}$ are the zeros. Since $f$ undergoes a $2 \pi$ phase change as $u$ encircles $b_{\alpha}$, the factor $\left(u-b_{\alpha}\right)$ describes a vortex at $\mathbf{b}_{\alpha}, b_{\alpha}=(\hat{\mathbf{x}}+i \hat{\mathbf{y}}) \cdot \mathbf{b}_{\alpha}$. If $Q$ extends to infinite, $f$ will be an infinite product. The most general form of $\Psi$ within the lowest Landau level is then $\Psi(x, y, z)=C(z) \prod_{\alpha}\left(u-b_{\alpha}(z)\right) e^{-r^{2} /\left(2 a_{\perp}^{2}\right)}$, or

$$
\Psi(x, y, z)=f(z) \phi(x, y ; z), \quad \int \mathrm{d} \mathbf{r}|\phi|^{2}=1
$$

where $\phi(x, y ; z)=\bar{\phi}(x, y ; z) / D(z)$

$$
\bar{\phi}(x, y ; z)=e^{-r^{2} /\left(2 a_{\perp}^{2}\right)} \prod_{\alpha=1}\left(u-b_{\alpha}(z)\right),
$$

and $D^{2}=\int \mathrm{d} \mathbf{r}|\bar{\phi}|^{2}$ is the normalization constant for $\phi$. The number constraint now becomes $\int \mathrm{d} z|f|^{2}=N$. Noting that within LLL,

$$
\int \Psi^{*} L_{z} \Psi=\hbar \int\left[\left(r / a_{\perp}\right)^{2}-1\right]|\Psi|^{2},
$$

and eq.(4) becomes

$$
\mathcal{K}(z)=\left(-\tilde{\mu}(z)+\hbar\left(\omega_{\perp}-\Omega\right)\left\langle r^{2}\right\rangle_{\phi}\right) f^{2}+\frac{g}{2} I_{\phi} f^{4}
$$

where $\tilde{\mu}(z)=\mu-\hbar \Omega-\frac{1}{2} M \omega_{z}^{2} z^{2},\left\langle r^{2}\right\rangle_{\phi}=\int r^{2}|\phi|^{2} \mathrm{~d} \mathbf{r}$ and $I_{\phi}=\int \mathrm{d} \mathbf{r}|\phi|^{4}$. The minimization is performed by varying $\left\{b_{\alpha}\right\}$. To evaluate $\left\langle r^{2}\right\rangle$ and $I_{\phi}$, we note that

$$
|\bar{\phi}|^{2}=e^{-\mathcal{H}}, \quad \mathcal{H}=r^{2} / a^{2}-2 \sum_{\alpha} \ln \left|\mathbf{r}-\mathbf{b}_{\alpha}\right| .
$$

The quantity $\mathcal{H}$ in eq.(9) is precisely the energy of a charge $q=-1$ particle in two dimension interacting with a uniform positive charge background $\rho_{b}=\pi / a^{2}$, and a set of $q=-1$ negative charges located at $\left\{\mathbf{b}_{\alpha}\right\}$. From eq.(9), we have

$$
\nabla_{\perp}^{2} \mathcal{H}=4 \pi\left(\left(\pi a^{2}\right)^{-1}-\sum_{\alpha} \delta\left(\mathbf{r}-\mathbf{b}_{\alpha}\right)\right),
$$

which is the Gauss's law for the 2D charged system. If $\left\{\mathbf{b}_{\alpha}\right\}$ forms an infinite regular lattice, $\mathbf{b}_{n_{1}, n_{2}}=$ $n_{1} \mathbf{c}_{1}+n_{2} \mathbf{c}_{2}, \quad\left(n_{1}, n_{2}\right.$ integers $)$, then $\sum_{\alpha} \delta\left(\mathbf{r}-\mathbf{b}_{\alpha}\right)=$ $v^{-1} \sum_{\mathbf{K}} e^{i \mathbf{K} \cdot \mathbf{r}}$, where $v=\left|\mathbf{c}_{1} \times \mathbf{c}_{2}\right|$ is the size of the unit cell, and $\mathbf{K}=\ell_{1} \mathbf{K}_{1}+\ell_{2} \mathbf{K}_{2}\left(\ell_{1}, \ell_{2}\right.$ integers $)$ are the reciprocal lattice vectors; $\left(\mathbf{K}_{1}=(2 \pi / v) \mathbf{c}_{2} \times \hat{\mathbf{z}}, \mathbf{K}_{2}=\right.$ $\left.(2 \pi / v) \hat{\mathbf{z}} \times \mathbf{c}_{1}\right)$. Eq.(10) and (9) then become

$$
\begin{aligned}
\nabla_{\perp}^{2} \mathcal{H} & =\frac{4}{\sigma^{2}}-\frac{4 \pi}{v} \sum_{\mathbf{K} \neq 0} \cos \mathbf{K} \cdot \mathbf{r}, \quad \frac{1}{\sigma^{2}}=\frac{1}{a_{\perp}^{2}}-\frac{\pi}{v}, \\
|\bar{\phi}|^{2} & =e^{-(r / \sigma)^{2}} \prod_{\mathbf{K} \neq 0} e^{-\zeta_{\mathbf{K}} \cos \mathbf{K} \cdot \mathbf{r}}, \quad \zeta_{K}=\frac{4 \pi}{v \mathbf{K}^{2}} .
\end{aligned}
$$

The Gaussian in eq.(12) corresponds to replacing the vortex density $\sum_{\alpha} \delta\left(\mathbf{r}-\mathbf{b}_{\alpha}\right)$ in eq.110) by its average $1 / v$. In the $2 \mathrm{D}$ electrostatics analog, it corresponds to reducing the uniform positive background by the average density of the discrete negative charges. Since the renormalized background is less confining for the negative unit charge at $u$, we have $\sigma>a_{\perp}$. Eq.(11) also requires $v>v^{*}=\pi a_{\perp}^{2}$, for otherwise $\phi$ will not be normalized. For hexagonal lattice, this means that the distance $c$ between neighboring vortices can not be shorter than $(2 \pi / \sqrt{3})^{1 / 2} a_{\perp}$.

The angular dependence of $|\bar{\phi}|^{2}$ is contained in the product in eq.(12). Since $v \mathbf{K}^{2}$ does not depend on the length of the basis vector $\mathbf{c}_{\alpha}$ but only on the lattice type (i.e. square or hexagonal), the product in eq.(12) determines mainly the lattice structure, and is less important in determining the overall density profile of the system. We shall therefore proceed by replacing the vortex density in eq.10) by its average, (referred to as "averaged vortex approximation", and come back later to consider the effect of the product in eq.(12). We shall see that the results of the averaged vortex approximation are intact except for changes of numerical factors (of order 1).

Averaged vortex approximation: Keeping only the Guassian in eq.(12), the normalized function $\phi$ in eq.(5) becomes $\phi(\mathbf{r})=\frac{1}{\pi \sigma^{2}} e^{-r^{2} / \sigma^{2}}$. Eq. (8) then becomes

$$
\mathcal{K}(z)=-\left[\tilde{\mu}(z)-\frac{\hbar\left(\omega_{\perp}-\Omega\right)}{\left(a_{\perp} / \sigma\right)^{2}}\right] f^{2}+\frac{\hbar \omega_{\perp} a_{s c} f^{4}}{\left(\sigma / a_{\perp}\right)^{2}}
$$

The optimal $\sigma$ and $f$ are

$$
\left[\frac{\sigma(z)}{a_{\perp}}\right]^{2}=\frac{\tilde{\mu}(z)}{3 \hbar\left(\omega_{\perp}-\Omega\right)}, \quad a_{s c} f^{2}(z)=\frac{[\tilde{\mu}(z)]^{2}}{9 \hbar^{2} \omega_{\perp}\left(\omega_{\perp}-\Omega\right)} .
$$


Since $\sigma \leq a_{\perp}$, the maximum extent of the cloud along $z$ satisfying this condition (denoted as $R_{z}$ ) is given by $3 \hbar\left(\omega_{\perp}-\Omega\right)=\tilde{\mu}\left(R_{z}\right)$. Note that within the average vortex approximation, $f(z)$ must vanish for $|z|>R_{z}$ in order to keep $\Psi$ normalized. We then have

$$
\tilde{\mu}(z)=\frac{1}{2} M \omega_{z}^{2}\left(R_{z}^{2}-z^{2}\right)+3 \hbar\left(\omega_{\perp}-\Omega\right) .
$$

The number condition $N=\int f^{2} \mathrm{~d} z$ then gives

$$
\frac{R_{z}}{a_{z}}=\left[\frac{135 N}{4}\left(\frac{a_{s c}}{a_{z}}\right)\left(\frac{\omega_{\perp}}{\omega_{z}}\right)^{2}\left(1-\frac{\Omega}{\omega_{\perp}}\right)\right]^{1 / 5}(1+. .)
$$

where $(1+\ldots)=\left(1-3 x+O\left(x^{2}\right)+..\right), x=\left[\left(\frac{\omega_{\perp}}{\omega_{z}}\right)^{1 / 5}(1-\right.$ $\left.\left.\frac{\Omega}{\omega_{\perp}}\right)^{3 / 5}\right] /\left[\frac{135 N}{4} \frac{a_{s c}}{a_{z}}\right]^{2 / 5}$. As we shall see, $x<<1$ for typical experimental parameters and can be ignored. From eq.(11) and (14), the size of the unit cell is given by

$$
v(z)=\pi a_{\perp}^{2}\left(1+\frac{6\left(\omega_{\perp}-\Omega\right)}{\omega_{z}} \frac{a_{z}^{2}}{R_{z}^{2}-z^{2}}\right) .
$$

In the fast rotating limit, $\Omega \rightarrow \omega_{\perp}$, eq.(17) is essentially the usual relation $n_{v}=2 \Omega /(h / M)$ for estimating the vortex density but in a more detailed form. (The connection is easily seen since $n_{v}=1 / v$, and $\pi a_{\perp}^{2}=\pi M \omega_{\perp} / \hbar \approx$ $2 M \Omega / h$. Note also that even though $\sigma^{2}$ shrinks quadratically as $z$ increases (eq.(14)), $v(z)$ (and hence the spacing between vortices) changes very little except near the tips of the condensate at $z \approx \pm R_{z}$.

A useful quantity is $\left(\pi \sigma^{2} / v\right)_{o} \equiv\left[\pi \sigma^{2} / v\right]_{z=0}$, the number of vortices inside an area $\pi \sigma^{2}$ at the center cross section (i.e. $z=0$ ). From eq.(14) and (17), we have

$$
\left(\frac{\pi \sigma^{2}}{v}\right)_{o}=\left(\frac{\pi a_{\perp}^{2}}{v-\pi a_{\perp}^{2}}\right)_{o}=\frac{\omega_{z}}{6\left(\omega_{\perp}-\Omega\right)}\left(\frac{R_{z}^{2}}{a_{z}^{2}}\right)
$$

Combining eq.(18) and eq.(16), we can express the equilibrium frequency $\Omega$ and $R_{z}$ in terms of the number of vortices in the center cross section,

$$
\begin{gathered}
\left(1-\frac{\Omega}{\omega_{\perp}}\right)=\left(\frac{v}{6 \pi \sigma^{2}}\right)_{o}^{5 / 3}\left(\frac{\omega_{z}}{\omega_{\perp}}\right)^{1 / 3}\left[\frac{135 N}{4}\left(\frac{a_{s c}}{a_{z}}\right)\right]^{2 / 3} \\
\frac{R_{z}}{a_{z}}=\left[\frac{135 N}{4}\left(\frac{a_{s c}}{a_{z}}\right)\left(\frac{\omega_{\perp}}{\omega_{z}}\right)\left(\frac{v}{6 \pi \sigma^{2}}\right)_{o}\right]^{1 / 3}+. .
\end{gathered}
$$

From eq.14) and (16), we find that the

$$
\sigma_{o}^{2} / a_{\perp}^{2}=(45 N / 8)\left(a_{s c} / a_{z}\right)\left(\omega_{\perp} / \omega_{z}\right)\left(a_{z} / R_{z}\right)^{3}
$$

The quantities $\Omega$ and $R_{z}$ for systems with different particle number and vortex numbers are shown in Table I. Finally, we note that the total angular momentum eq.(7) of the system is $\left\langle L_{z}\right\rangle=\hbar \frac{6 N}{7}\left(\frac{\pi \sigma^{2}}{v}\right)$. The total energy is $E=\mathcal{K}+\Omega\left\langle L_{z}\right\rangle+\mu N$. To the leading term in $N$, we have $E=\frac{\omega_{\perp}}{243}\left(\frac{\omega_{z}}{\omega_{\perp}}\right)^{3}\left(1-\frac{\Omega}{\omega_{\perp}}\right)^{-2}\left(\frac{a_{z}}{a_{s c}}\right)\left(R_{z} / a_{z}\right)^{7}$, hence $E \propto N^{7 / 5}$.

Mixing of higher Landau levels and the validity of Thomas-Fermi approximation (TFA): The validity of TFA requires requires $\left(\hbar^{2} / 2 M\right)\left|\nabla_{z} \Psi\right|^{2}<<\mu(z)|\Psi|^{2}$, which is $\hbar^{2} /\left(2 M R_{z}^{2}\right)<<\mu$ or $\left(R^{2} / a_{\perp}\right)^{4}>>1$. This is easily satisfied for the range of parameters listed in Table I. Mixing of higher Landau level will be unimportant if the interaction energy density $\hbar \omega_{\perp}\left(a_{\perp} / \sigma\right)^{2} a_{s c} f^{4}$ is much less than that of higher Landau level $\hbar \omega_{\perp} f^{2}$, or $\Gamma \equiv\left(a_{\perp} / \sigma\right)^{2} a_{s c} f^{2}<<1$. From eq.(14), we have $\Gamma=\tilde{\mu}(z=0) /\left(3 \hbar \omega_{\perp}\right)$, or $\Gamma=\frac{\omega_{z}}{6 \omega_{\perp}}\left(R_{z} / a_{z}\right)^{2}$. The values of $\Gamma$ for a variety of external parameters are shown in Table I. Although the recent MIT experiment [1] is not yet in the LLL regime, it is not too far away. It is clear from Table I that the LLL regime can be reached within the capability of current technology. Note also that as $\Omega \rightarrow \omega_{\perp}$ and the system is driven more toward the LLL regime. However, $R_{z} / a_{z}$ also decreases. As $R_{z}$ becomes very close to $a_{z}$, Thomas-Fermi approximation will not be valid and a more accurate treatment along $z$ is needed. The present treatment is valid for the frequencies $\Omega$ such that $R_{z} / a_{z}>1$, which is satisfied for the cases listed.

Beyond averaged vortex approximation: A full calculation of the quantities $\left\langle r^{2}\right\rangle_{\phi}$ and $I_{\phi}$ in eq.(4) requires considering the "structural" product in eq.(12). We shall show that in the limit $\Omega \rightarrow \omega_{\perp}$, the major effect of the structural product is to stabilize the hexagonal lattice over the square lattice. It does not alter the result $\left\langle r^{2}\right\rangle_{\phi}$ of the averaged vortex approximation, but changes $I_{\phi}$ from $1 /\left(2 \pi \sigma^{2}\right)$ to $\alpha /\left(2 \pi \sigma^{2}\right)$ with $\alpha \sim 1$. Thus, all results of the simple average vortex approximation remain.

To prove the above statements, we recall that $e^{-\alpha \cos \theta}=\sum_{n=0,1,2, . .}(-1)^{n} I_{n}(\alpha) e^{i n \theta}$, where $I_{n}(\alpha)$ are the modified Bessel functions. Eq.(12) then becomes $|\bar{\phi}|^{2}=e^{-r^{2} / \sigma^{2}} \prod_{\mathbf{K} \neq 0}\left(\sum_{n_{\mathbf{K}}}(-1)^{n_{\mathbf{K}}} I_{n_{\mathbf{K}}}\left(\zeta_{\mathbf{K}}\right) e^{i n_{\mathbf{K}} \mathbf{K} \cdot \mathbf{r}}\right)$, or

$$
|\bar{\phi}|^{2}=e^{-r^{2} / \sigma^{2}} \sum_{\left[n_{\mathbf{K}}\right]}^{\prime} \Lambda\left[n_{\mathbf{K}}, \zeta_{\mathbf{K}}\right] e^{i \mathbf{P}\left[n_{\mathbf{K}}\right] \cdot \mathbf{r}},
$$

$$
\Lambda\left[n_{\mathbf{K}}, \zeta_{\mathbf{K}}\right]=\prod_{\mathbf{K} \neq 0}(-1)^{n_{\mathbf{K}}} I_{n_{\mathbf{K}}}\left(\zeta_{\mathbf{K}}\right), \quad \mathbf{P}\left[n_{\mathbf{K}}\right]=\sum_{\mathbf{K}} \mathbf{K} n_{\mathbf{K}},
$$

where $\sum^{\prime}$ in eq.(22) means the $\mathbf{K}=0$ term is excluded, and $\left[n_{\mathbf{K}}, \zeta_{\mathbf{K}}\right]$ denotes the entire sets $\left\{n_{\mathbf{K}}\right\}$ and $\left\{\zeta_{\mathbf{K}}\right\}$. The normalized function $|\phi|^{2}$ is then

$$
\begin{gathered}
|\phi|^{2}=\frac{e^{-r^{2} / \sigma^{2}}}{\pi \sigma^{2}} \frac{\prod_{\mathbf{K} \neq 0} e^{-\zeta_{\mathbf{K}} \cos \mathbf{K} \cdot \mathbf{r}}}{Z_{\sigma^{2}}\left[\zeta_{\mathbf{K}}\right]}, \\
Z_{\sigma^{2}}\left[\zeta_{\mathbf{K}}\right]=\sum_{\left[n_{\mathbf{K}}\right]} \Lambda\left[n_{\mathbf{K}}, \zeta_{\mathbf{K}}\right] e^{-\sigma^{2} P\left[n_{\mathbf{K}}\right]^{2} / 4} .
\end{gathered}
$$


We then have $\left\langle r^{2}\right\rangle_{\phi}=\sigma^{2} \gamma, \int|\phi|^{4}=\alpha /\left(2 \pi \sigma^{2}\right)$, where $\gamma=Z_{\sigma^{2}}\left[\zeta_{\mathbf{K}}\right]^{-1} \sum_{\left[n_{\mathbf{K}}\right]} \Lambda\left[n_{\mathbf{K}}, \zeta_{\mathbf{K}}\right] e^{-\left(\sigma P\left[n_{\mathbf{K}}\right] / 2\right)^{2}}$ $\left(1-\frac{\sigma^{2} P\left[n_{\mathbf{K}}\right]^{2}}{4}\right)$, and $\alpha=Z_{\sigma^{2} / 2}\left[2 \zeta_{\mathbf{K}}\right] Z_{\sigma^{2}}\left[\zeta_{\mathbf{K}}\right]^{-2}$. As $\Omega \rightarrow \omega_{\mathcal{L}}, \sigma^{2} P\left[n_{\mathbf{K}}\right]^{2}>>1$ for all $\mathbf{P}\left[n_{\mathbf{K}}\right] \neq 0$. Only terms in eq.(25) with $\sum_{\mathbf{K}} \mathbf{K} n_{\mathbf{K}}=0$ are important. This implies $\gamma=1,\left\langle r^{2}\right\rangle_{\phi}=\sigma^{2}$, and

$$
\begin{gathered}
\alpha=\tilde{Z}\left[2 \zeta_{\mathbf{K}}\right] /\left(\tilde{Z}\left[\zeta_{\mathbf{K}}\right]\right)^{2}, \\
\tilde{Z}\left[\zeta_{\mathbf{K}}\right]=\sum_{n_{\mathbf{K}}}^{\prime \prime} \prod_{\mathbf{K} \neq 0}(-1)^{n_{\mathbf{K}}} \tilde{I}_{n_{\mathbf{K}}}\left(\zeta_{\mathbf{K}}\right),
\end{gathered}
$$

where $\sum^{\prime \prime}$ means summing over $\left\{n_{\mathbf{K}}\right\}$ with such that $\sum_{\mathbf{K}} \mathbf{K} n_{\mathbf{K}}=0$.

To evaluate $\alpha$, we rewrite $\tilde{Z}$ as $\tilde{Z}\left[\zeta_{\mathbf{K}}\right]=$ $W\left[\zeta_{\mathbf{K}}\right] \sum_{\left[n_{\mathbf{K}}\right]}^{\prime \prime} \prod_{\mathbf{K} \neq 0}(-1)^{n_{\mathbf{K}}} \tilde{I}_{n_{\mathbf{K}}}\left(\zeta_{\mathbf{K}}\right)$, where $W\left[\zeta_{\mathbf{K}}\right]=$ $\prod_{\mathbf{K} \neq 0} I_{0}\left(\zeta_{\mathbf{K}}\right)$, and $\tilde{I}_{n}(\zeta)=I_{n}(\zeta) / I_{0}(\zeta)$. Let us define $\zeta_{o}=\zeta_{\mathbf{K}_{o}}$, where $\mathbf{K}_{o}$ is the shortest reciprocal lattice vector. Since $\zeta_{o}=\sqrt{3} /(2 \pi)$ and $1 / \pi$ for hexagonal and square lattice respectively, and since $I_{n}(\zeta)=$ $\sum_{k=0}^{\infty} \frac{(\zeta / 2)^{n+2 k}}{k !(n+k) !}$, the sums in eq.(26) are fast convergent. $\operatorname{Regarding} \tilde{Z}$ formally as a power series of $\zeta_{o}$, we have for the hexagonal lattice, $W\left[\zeta_{\mathbf{K}}\right]=1+\left(3 \zeta_{o}^{2} / 2\right) \beta_{1}+.$. , and

$$
\tilde{Z}\left[\zeta_{\mathbf{K}}\right]=W\left[\zeta_{\mathbf{K}}\right]\left(1+\frac{3 \zeta_{o}^{2}}{4} \beta_{1}-\frac{\zeta_{o}^{3}}{4} \beta_{2}+\ldots\right)
$$

where $\beta_{n}=\sum_{\ell_{1}=1}^{\infty} \sum_{\ell_{2}=0}^{\infty}\left(\ell_{1}^{2}+\ell_{2}^{2}+\sqrt{3} \ell_{1} \ell_{2}\right)^{-2 n}$. The $\zeta_{o}^{2}$ term in $W$ comes from expanding $I_{o}\left(\zeta_{\mathbf{K}}\right)$ in $\zeta_{o}$ to second order in $\zeta_{o}$. The $\zeta_{o}^{2}$ term in eq. (28) comes from pairs of $\tilde{I}_{1}$ terms in $\tilde{Z}$. The factor $\beta_{1}$ is the result of summing over all such pairs. The $\zeta_{o}^{3}$ term in eq.(28) comes from triplets of vectors of the same length in eq.(27). These triplets give rise to a negative sign which lowers the energy of the system. The factor $\beta_{2}$ is the result of summing all these isosceles triplets. Using eq.(28) and eq.(26), we have $\alpha=1+\frac{9}{2} \zeta_{o}^{2} \beta_{1}-\frac{1}{4} \zeta_{o}^{3} \beta_{2}+\ldots$ to orders less than $\zeta_{o}^{4}$. The result is $\alpha=1.38,\left(\beta_{1}=1.218, \beta_{2}=1.001\right.$, $\left.\zeta_{o}=0.276\right)$.

Repeating the same calculation for the the square lattice. We have, to the lowest order in $\zeta_{o}, W\left[\zeta_{\mathbf{k}}\right]=1+$ $\zeta_{o}^{2} \gamma_{1}+. ., \tilde{Z}\left[\zeta_{\mathbf{K}}\right]=W\left[\zeta_{\mathbf{k}}\right]\left(1+\frac{1}{2} \zeta_{o}^{2} \gamma_{1}+\ldots\right)$, where $\gamma_{1}=\sum_{\ell_{1}=1}^{\infty}$ $\sum_{\ell_{2}=0}^{\infty}\left(\ell_{1}^{2}+\ell_{2}^{2}\right)^{-2}=1.506$, which gives $\alpha=1+3 \zeta_{o}^{2} \gamma_{1}+.$. to orders less than $\zeta_{o}^{4}$. The result is $\alpha=1.45$, since $\zeta_{o}=0.318$. The square lattice has energy higher than the hexagonal one because it has no negative triplets terms.

Final Remarks: We have shown that as the number of vortices increases, a Bose-Einstein condensate will become quantum Hall like. A natural question is what happens if the angular momentum of the system keeps increasing. We have seen that $R_{z}$ decreases as angular momentum increases. Eventually, it will reduce to the point where only one harmonic state in the z-direction will be occupied. In such "ultra-fast" rotating limit, fluctuations effects in the $x y$ plane will be important and one has to go beyond mean field treatment. The discussion of the ultra-fast limit will be presented elsewhere.

I thank Wolfgang Ketterle for sending me his preprint before its publication, and Mehmet Oktel for discussions. This work is supported by the NASA Grant NAG8-1441, and the NSF Grants DMR-9807284, DMR-0071630.

\begin{tabular}{|l|l|l|l||l|l|l|l|}
\hline & $\mathrm{N}\left(10^{6}\right)$ & $\omega_{\perp} / 2 \pi$ & $\omega_{z} / 2 \pi$ & $\pi \sigma^{2} / v$ & $1-\Omega / \omega_{\perp}$ & $R_{z} / a_{z}$ & $\Gamma$ \\
\hline $\mathrm{a}$ & 50 & 84 & 20 & 40 & 0.64 & 6.8 & 1.85 \\
\hline $\mathrm{b}$ & 50 & 1000 & 10 & 40 & 0.179 & 10.9 & 0.198 \\
\hline $\mathrm{c}$ & 50 & 1000 & 10 & 100 & 0.038 & 9.3 & 0.146 \\
\hline $\mathrm{d}$ & 5 & 84 & 20 & 40 & 0.13 & 4.6 & 0.86 \\
\hline $\mathrm{e}$ & 5 & 1000 & 10 & 40 & 0.039 & 7.4 & 0.092 \\
\hline $\mathrm{f}$ & 5 & 1000 & 10 & 100 & 0.008 & 6.38 & 0.06 \\
\hline
\end{tabular}

Caption: Row (a) corresponds to the parameters of the recent MIT experiment [i]. We have counted the vortices as follows. The Gaussian density profile $|\phi|^{2}$ in eq.(12) drops by a factor of 100 from the center to a radius $r=2 \sigma$. If there are 160 vortices within a radius of $2 \sigma$, then $\pi \sigma^{2} / v=40$. To remain in the lowest Landau level (LLL), one needs $\Gamma<<1$. The parameters of recent MIT experiment [1] is not yet in the LLL regime. However, the latter can be reached by changing the parameters as indicated in case (b) to (f). For all cases, the quantity $x$ defined after eq.(16) is $\sim 10^{-3}$.

[1] J.R. Abo-SHaeer, C. Raman, J. M. Vogels, and W. Ketterle, to appear in Science.

[2] K.W. Madison, F. Chevy, W. Wohlleben and J. Dalibard, Phys. Rev. Lett. 84, 806 (2000), F. Chevy, K. Madison, and J. Dalibard, Phys. Rev. Lett. 85, 2223 (2000), and F. Chevy, K.W. Madison, V. Bretin, and J. Dalibard, condmat/0104218.

[3] At criticality, $\Omega=\omega_{\perp}$, eq.(1) is identical to the free energy of type-II superconductor. The two problems are different because the constraint $N=\int|\Psi|^{2}$ does not exist in typeII superconductors, and the Maxwell equation in type-II superconductors does not exist in Bose gases.

[4] N.K. Wilkin and J.M.F. Gunn, Phys. Rev. Lett. 84, 6 (2000). N. K. Wilkin, J. M. F. Gunn, and R. A. Smith, Phys. Rev. Lett. 80, 2265 (1998).

[5] Since eq.(11) is a second order differential equation, $\mathcal{H}$ has a term linear in $\mathbf{r}$. Such term vanishes if the origin is a point of symmetry, which we assume for simplicity. 